\section{Need For HBV Cure: Persistent Risk For Subsequent New And Recurrent HCC Even After A Decade Of Successful Anti-HBV Therapy And Initial Tumor Ablation}

\section{Abstract}

Since 1998 with the highly effective nucleos(t)ide analogues for Hepatitis B virus (HBV), we have witnessed successful viral suppression that has led to the delay or prevention of the development of hepatocellular carcinoma (HCC). Reduced incidence of HCC with anti-HBV treatment has been documented with lamivudine, entecavir and tenofovir. A number of investigators have observed that after the initial tumor was ablated, anti-HBV treatment could prevent or reduce the recurrent or subsequent new HCC. Nonetheless, as reported earlier, we are observing the persistent risk for HCC in patients in spite of successful viral suppression for over a decade with anti-HBV therapy. In this report, we present three cases who underwent initial tumor ablation and maintained negative HBV DNA with anti-HBV therapy and yet developed subsequent new or recurrent $\mathrm{HCC}$ at the intervals of 5,10,11 and 15 years. These persistent risks for new and recurrent $\mathrm{HCC}$ are attributed to the incomplete control of HBV due to the presence of cccDNA in the host's hepatocytes. Current antiviral treatment can achieve a functional cure (suppression of HBV replication) while a complete cure of eliminating the cccDNA has not been possible. Therefore, even though HBV is not actively replicating, cccDNA remains in the nucleus of the hepatocytes and continues hepatocarcinogenic processes including HBV and host DNA integration. There is a need for HBV cure drugs.

Keywords: HBV HCC; Antiviral therapy; Nucleos(t)ie analogues; HCC risk; HBV cure; Hepatocarcinogenesis; ccc DNA

Received: April 25, 2018; Accepted: May 08, 2018; Published: May 12, 2018

\author{
Brianna Shinn ${ }^{1}$, \\ Charles Kistler ${ }^{1}$, \\ Christopher Roth ${ }^{2}$ and \\ Hie Won Hann ${ }^{1,3 *}$
}
1 Division of Gastroenterology and Hepatology, Sidney Kimmel Medical College, Thomas Jefferson University Hospital, Philadelphia, USA
2 Department of Radiology, Sidney Kimmel Medical College, Thomas Jefferson University Hospital, Philadelphia, USA 3Liver Disease Prevention Center3, Sidney Kimmel Medical College, Thomas Jefferson University Hospital, Philadelphia, USA

\section{*Corresponding author: Hie-Won Hann \\ ほ hie-won.hann@jefferson.edu}

Liver Disease Prevention Center, Sidney Kimmel Medical College, Thomas Jefferson University Hospital, Philadelphia, USA.

Tel: $215-955-5806$

Fax: 215-955-0770

Citation: Shinn B, Kistler C, Roth C, Hann HW (2018) Need For HBV Cure: Persistent Risk For Subsequent New And Recurrent HCC Even After A Decade Of Successful AntiHBV Therapy And Initial Tumor Ablation. Arch Cancer Res. Vol.6 No.2:6

\section{Introduction}

Chronic hepatitis B virus (HBV) places patients at an increased risk for the development of hepatocellular carcinoma (HCC) $[1,2]$. The available antiviral drugs (nucleos(t)ide analogues) are highly effective in suppressing the replication of the virus. Therefore, reduction of incidence of HBV-related HCC has been observed with antiviral drugs including lamivudine, entecavir and tenofovir [3-6]. However, despite the use of antiviral therapies, development of HCC in patients with chronic HBV has been reported. Most data regarding this persistent risk for HCC is seen in studies with short-term follow up of 4-5 years [7-10]. Much longer follow up studies of 20 years were reported lately $[11,12]$. The development of subsequent new or recurrent HCC after initial tumor ablation and viral suppression has not been reported. Here we present three cases which demonstrate the persistent risk for HCC after initial tumor ablation and decades of successful antiviral therapy.

\section{Case 1}

A 40-year-old Asian man was noted to be HBsAg (+) in 1987. At that time he had minimal symptoms including fatigue and he continued to drink alcohol socially. Additional labs at that time included ALT $400 \mathrm{IU} / \mathrm{L}, \mathrm{HBeAg}(+) /$ anti-HBe (-), HBV DNA (+) (qualitative value only) and AFP $5.3 \mathrm{ng} / \mathrm{ml}$. After discontinuing alcohol, the patient's ALT became normal a few months later. No anti-HBV medication was available at that time and he was conservatively followed with routine labs until 1991, after which 
he continued to follow up with his primary care physician only. In 2004, 17 years later, at age 57, the patient came back referred by his physician when a $4.0 \mathrm{~cm}$ liver mass concerning for HCC was detected in segment 8 on abdominal ultrasound (Figure 1). Imaging was also consistent with early cirrhotic changes with medial segment atrophy. At that time, laboratory results showed HBV DNA (+) (qualitative value only), ALT $59 \mathrm{IU} / \mathrm{L}$, platelets 213B/L, AFP $44.6 \mathrm{ng} / \mathrm{ml}$ and HBeAg (-)/anti-HBe (+). During the 17 year interval, he had spontaneously converted to anti-HBe. He underwent transarterial chemoembolization (TACE) followed by radiofrequency ablation (RFA) the next day following an

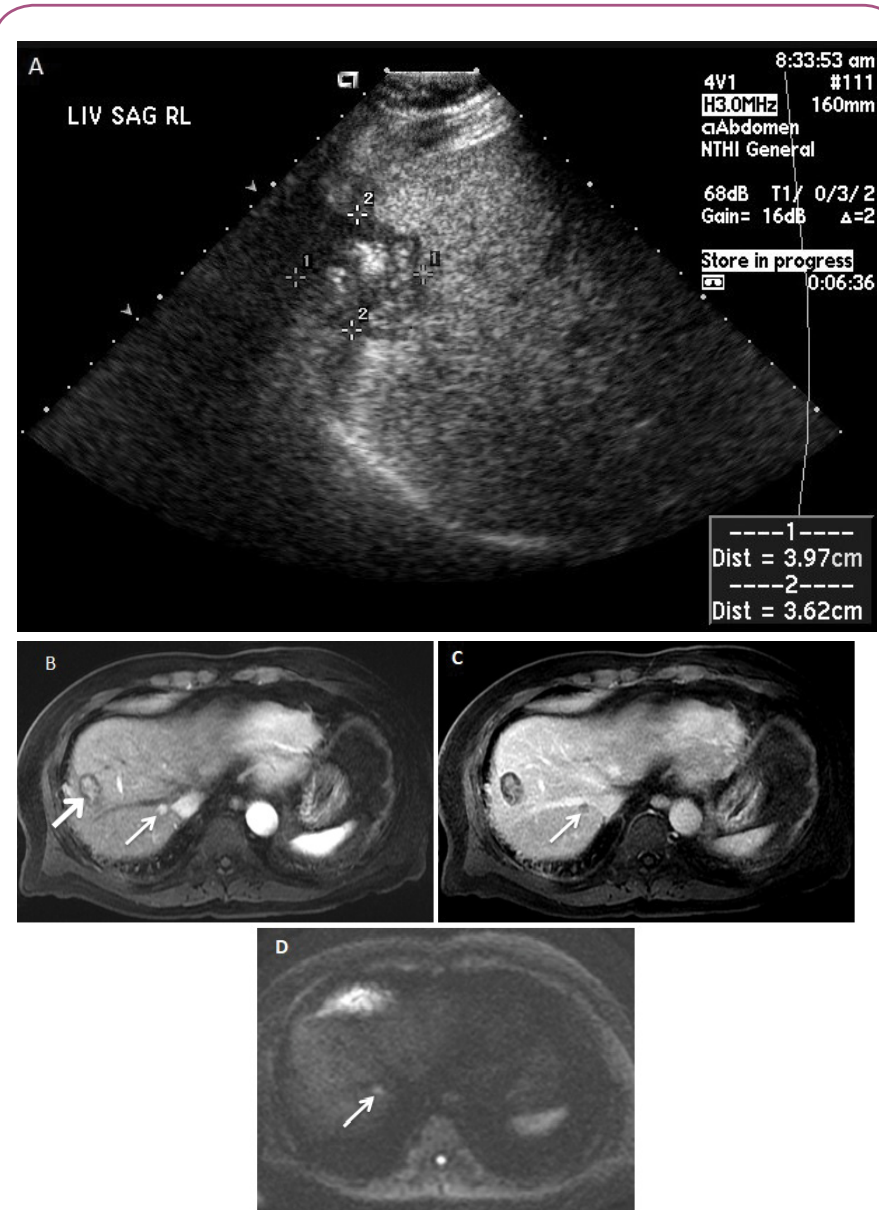

Figure 1 Case 1 (10/20/2004) (A) Longitudinal ultrasound image through the right hepatic lobe shows a $4 \mathrm{~cm}$ hypoechoic solid mass (calipers) with echogenic foci corresponding to hepatocellular carcinoma. Case 1 (3/31/2014) (B) 10 years later, following transarterial chemoembolization and the initiation of anti-HBV therapy, the post-contrast T1-weighted arterial-phase image reveals a $1 \mathrm{~cm}$ hyperenhancing lesion in hepatic segment 7 (arrow) and the residuum of the previously noted lesion following transarterial chemoembolization (thick arrow). (C) The T1-weighted portal venous-phase image shows that the hyperenhancing lesion demonstrates washout and capsule appearance, features that are diagnostic of hepatocellular carcinoma. (D) The diffusion-weighted image shows lesion hyperintensity (arrow) indicating diffusion restriction.

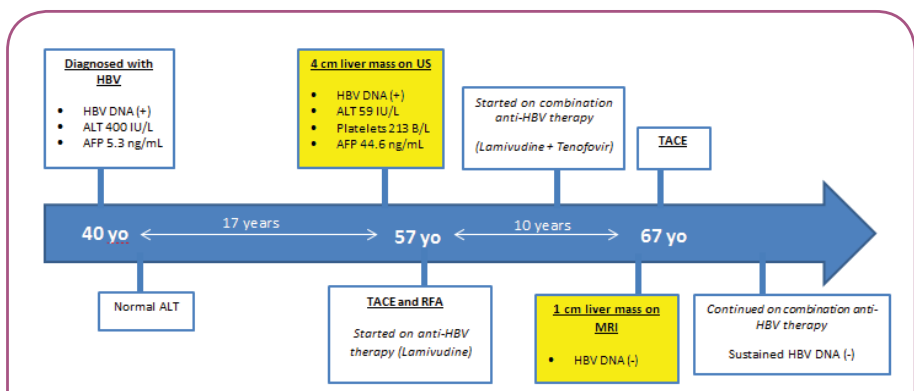

Case 1 Timeline of the first and a new HCC (highlighted).

established protocol at the time. He was also started lamivudine $150 \mathrm{mg}$ daily. HBV DNA became undetectable in a month and continued to remain undetectable throughout the follow up period. Tenofovir $300 \mathrm{mg}$ daily was added six years later as the combination therapy was preferred. In 2014, 10 years after the first tumor ablation, at age 67, on MRI a new $1.0 \mathrm{~cm}$ arterially enhancing lesion with washout appeared in segment 7 of the liver. It was compatible with HCC. He underwent successful TACE one month later. Since then, he has been on dual anti HBV therapy with lamivudine and tenofovir with sustained undetectable HBV DNA and close follow-up (Case 1).

\section{Case 2}

A 52-year-old Asian woman was first noted to be HBsAg (+) in 1987 during a routine examination. Her three children, all in their 20 s at the time, were also HBsAg (+), suggesting she had HBV infection for over 20 years by then. After 3 years of follow up at our institution her family moved in 1990 and she was lost to follow-up. In 2000, at age 65, she returned for a follow up. She had not received antiviral treatment in the meantime. Abdominal MRI showed a $2.5 \mathrm{~cm}$ mass in the anterior segment of the right lobe with arterial hyper enhancement and washout appearance, consistent for HCC (Figure 2). AFP was $6.7 \mathrm{ng} / \mathrm{ml}$ and HBV DNA was $1.75 \mathrm{pg} / \mathrm{ml}$. She was $\mathrm{HBeAg}(-) /$ anti-HBe(+) with albumin 4.0, ALT $29 \mathrm{IU} / \mathrm{L}$, total bilirubin 0.4 and platelets 122 period Liver was mildly nodular. After undergoing tumor ablation with RFA followed by TACE, the patient was started on antiviral therapy with lamivudine $150 \mathrm{mg}$ daily. HBV DNA became undetectable $(<0.002 \mathrm{pg} / \mathrm{ml})$ in 3 months. Four years later, HBV DNA was 44.65 $\mathrm{pg} / \mathrm{ml}$ and she was suspected to have developed lamivudine resistance and antiviral treatment was changed to tenofovir 300 mg daily. HBV DNA became undetectable six months later. In 2005, five years after the first tumor ablation, while continued on anti-HBV therapy with undetectable HBV DNA, a $1.1 \mathrm{~cm} \times 0.8 \mathrm{~cm}$ arterially enhancing lesion with washout appearance was noted near the treated site on abdominal MRI. The patient underwent TACE and continued anti-HBV therapy. In 2015, 10 years later at age 80 (15 years after the first tumor and with undetectable HBV DNA), an abdominal MRI again showed an $8.0 \mathrm{~mm}$ recurrent HCC near the previously treated site. The tumor was treated with microwave (MW) ablation. The patient, now age 83 years old, continues to have close follow-up with an undetectable HBV viral load. 

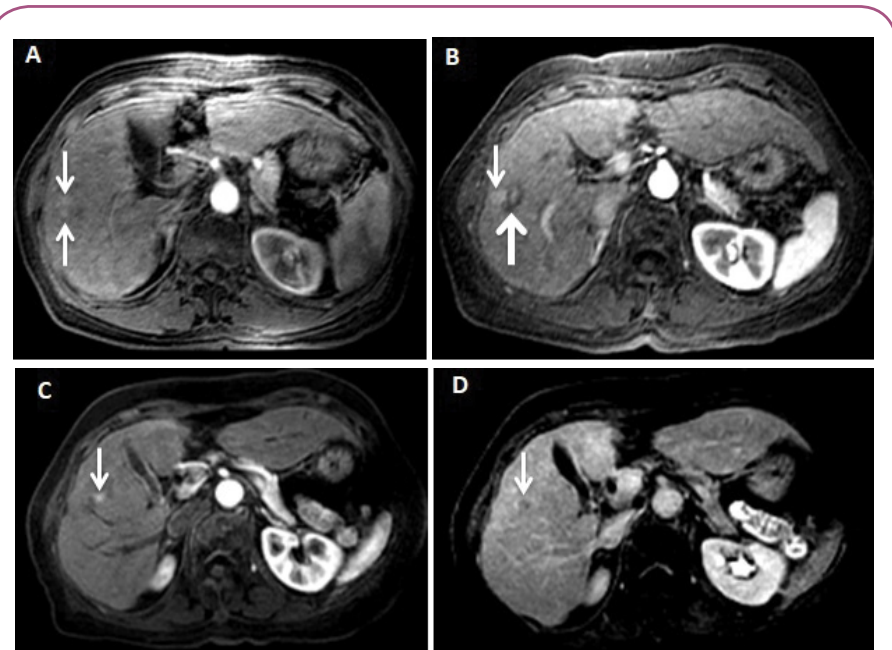

Figure 2 Case 2 (3/17/2000) (A)The arterial-phase postcontrast T1-weighted image reveals cirrhosis with nodularity and a hyper-enhancing lesion in hepatic segment 5 (arrows) with portal-phase washout (not shown) consistent with hepatocellular carcinoma, which was treated with radiofrequency ablation and transarterial chemoembolization. (B) The arterialphase post-contrast T1-weighted image from a study obtained 5 years later shows a new $1.1 \mathrm{~cm}$ hyperenhancing lesion (arrow) lateral to the ablated lesion (thick arrow) indicating recurrent tumor. (C) 10 years later following a second bout of transarterial chemoembolization treating the second lesion, the arterial-phase post-contrast T1-weighted image shows a third subcentimeter hyperenhancing lesion (arrow) with washout on the portal phase image (D) Arrow suggesting hepatocellular carcinoma, which was treated with microwave ablation.

\section{Case 3}

A 57-year-old Asian man was noted to be HBsAg (+) in 1996 . He denied family history of HBV. He had never received antiviral therapy. In 2006, at age 67, he developed bronchitis and had lab work performed which demonstrated elevated ALT of $98 \mathrm{IU} / \mathrm{L}$. A subsequent abdominal MRI showed a cirrhotic liver with 1.0 $\mathrm{cm}$ mildly hypervascular fat-containing lesion suspicious for HCC (Figure 3). Further work up showed HBV DNA $10.3 \times 106$ copies/ml, HBeAg (+), ALT $57 \mathrm{IU} / \mathrm{ml}$, and AFP $12.5 \mathrm{ng} / \mathrm{ml}$. He then came to our institution for further evaluation. The patient underwent RFA and was started on combination anti-HBV therapy with lamivudine $150 \mathrm{mg}$ and tenofovir $300 \mathrm{mg}$ daily, the preferred regimen for $\mathrm{HCC}$ at that time. Within six months of anti-HBV treatment, HBV DNA became undetectable. He was then followed by his family physician and had imaging studies every six to twelve months. In 2017, 11 years after the first tumor ablation, now at age 78 , he presented with an abnormal MRI which showed two liver lesions with imaging features diagnostic of HCC: a $3.0 \mathrm{~cm} \times 2.8 \mathrm{~cm}$ lesion near the gallbladder and another $2.0 \mathrm{~cm} \times 1.7 \mathrm{~cm}$ lesion in the posterior segment of the right lobe. Laboratory results showed AFP $24.4 \mathrm{ng} / \mathrm{ml}$, undetectable HBV DNA, ALT $19 \mathrm{IU} / \mathrm{L}$, platelets $125 \mathrm{~B} / \mathrm{L}$ and $\mathrm{HBeAg}(-) /$ anti-HBe (+). He underwent MW tumor ablation. One month later, his AFP decreased to $3.7 \mathrm{ng} / \mathrm{ml}$. and a recent follow up MRI eight months later showed no new concerning lesions (Case 3).

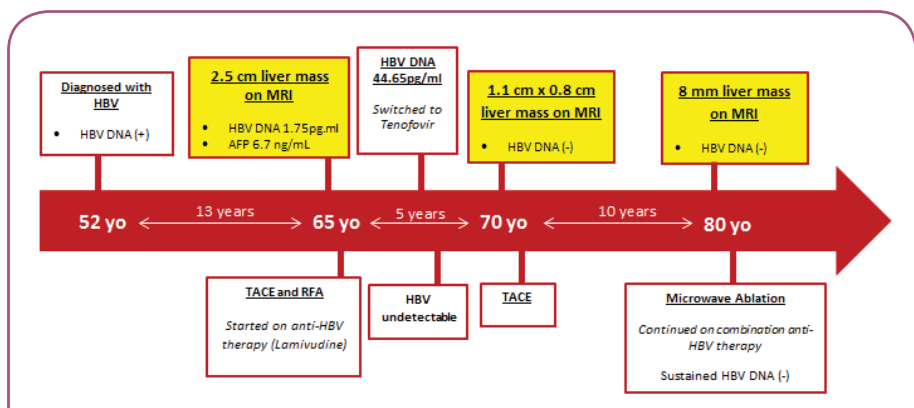

Case 2 Timeline of the first and recurrent HCC (highlighted).

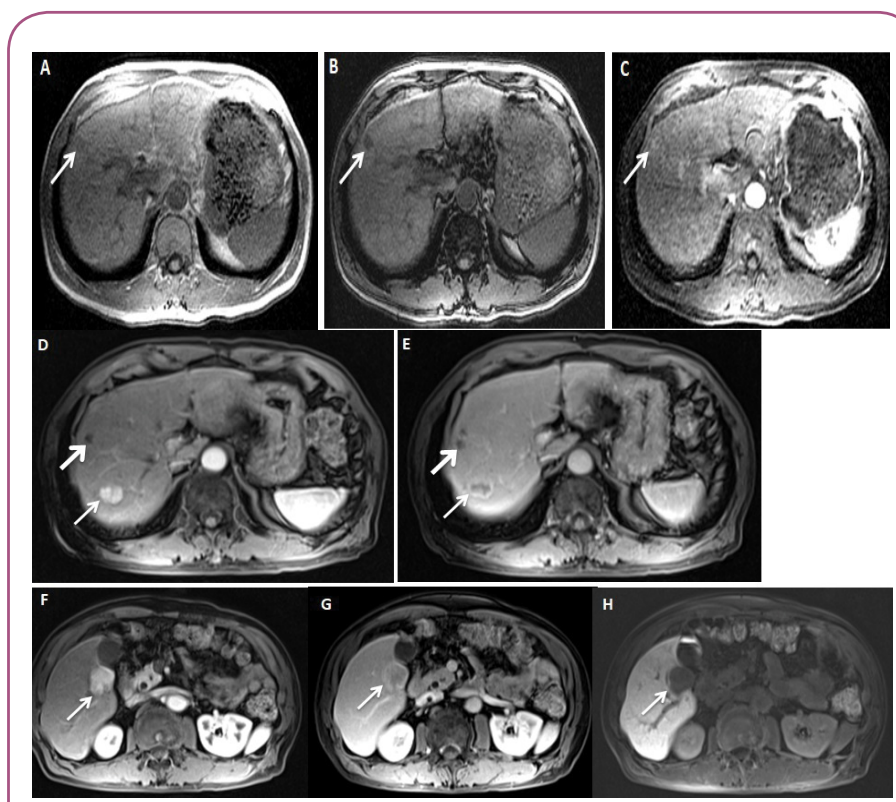

Figure 35 Case $3(1 / 30 / 2006)$ (A)The in-phase image shows a mildly hypointense lesion at the junction of segments 5 and 8 (arrow), which is more conspicuously hypointense on the out-of-phase image ( $B$, arrow), indicating the presence of lipid content characteristic of hepatocellular carcinoma with liver nodularity indicating cirrhosis. (C) There is faint hyperenhancement on the arterialphase post-contrast T1-weighted image (arrow). Case $3(4 / 3 / 2017)$ (D) 11 years following radiofrequency ablation and the initiation of anti-HBV therapy, a subsequent arterial-phase post-contrast T1-weighted image reveals a new hyperenhancing mass in segment 7 (arrow). (E) The portal phase image demonstrates washout and a capsule appearance (arrow) indicating recurrent hepatocellular carcinoma. The punctate hypointensity at the junction of hepatic segments 5 and 8 (thick arrow) corresponds to the lesion ablated 11 years earlier. (F) A second larger lesion is seen in segment 5 (arrow) exhibiting hyperenhancement on the arterialphase post-contrast T1-weighted image, washout and capsule appearance on the portal-phase image (G) and hypointensity on the hepatobiliary-phase post-contrast T1-weighted image $(\mathrm{H})$-all features consistent with hepatocellular carcinoma. 


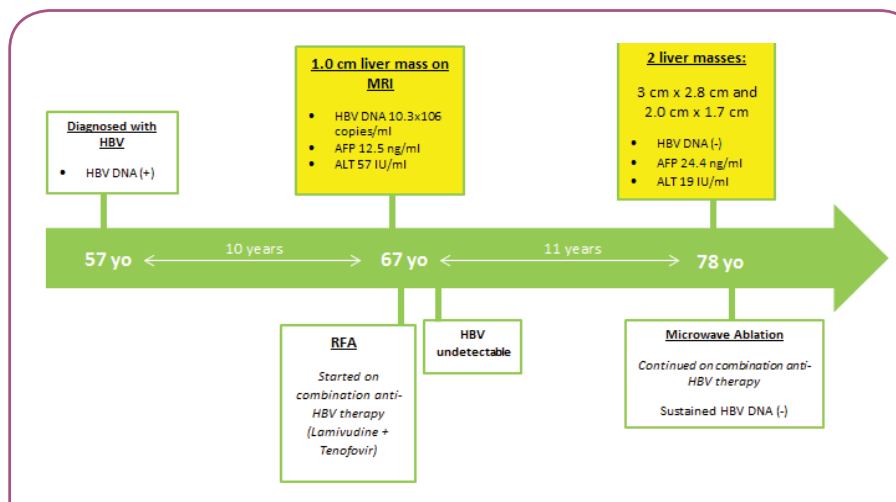

Case 3 Timeline of the first and new HCC (highlighted).

\section{Discussion}

Since the advent of anti-HBV therapy (nucleos(t)ide analogues), a reduced incidence of HCC has been well documented with lamivudine, entecavir and tenofovir. [3-6] On the other hand, despite HBV DNA at undetectable levels, some patients, especially those with cirrhosis were found to develop HCC. Most observations were derived from a relatively short follow-up period of four to five years [7-10]. The longest follow up study was presented by Dargan et al. and Yoo et al. [11,12]. In their study they followed patients on anti-HBV treatment for more than 20 years and found that the risk for HCC persisted even with successful clearance of HBV viremia. Several patients had been on anti-HBV therapy for greater than 18 years and yet developed HCC. These patients have had undetectable HBV DNA in serum over 10 years and maintained normal liver function. Furthermore, several studies have shown that patients following initial tumor ablation and on anti-HBV therapy have had no recurrent tumors [13-18]. Now we presented three cases that illustrate the persistent risk for subsequent new or recurrent HCC in spite of initial successful tumor ablation, continued anti-HBV therapy and sustained clearance of HBV DNA viremia for over ten years. The first patient we presented developed recurrent HCC ten years after the initial HCC diagnosis. The first tumor was successfully ablated and the patient was continued on anti-HBV therapy. The patient had an undetectable HBV DNA level for the ten years prior to the diagnosis of the second tumor. The second patient we presented had a recurrence of HCC five years after treatment for initial HCC. At the time of recurrent HCC, the patient had been on anti-HBV therapy and had an undetectable HBV DNA level. Another ten years after the first recurrence, the patient developed a second HCC recurrence while still on anti-HBV therapy with an undetectable HBV DNA level. The third patient we presented had a recurrent HCC eleven years after initial HCC diagnosis while maintained on anti-HBV therapy with successful viral suppression. The persistence of the risk for HCC in these cases is shown (Figure 4). To understand the persistent risk of HCC development despite clearance of HBV DNA, it is imperative to understand the HBV life cycle and the steps leading to hepatocarcinogenesis [19-24]. HBV is an enveloped partially double-stranded DNA virus. When the virus enters the hepatocytes, most of the viral DNA is converted

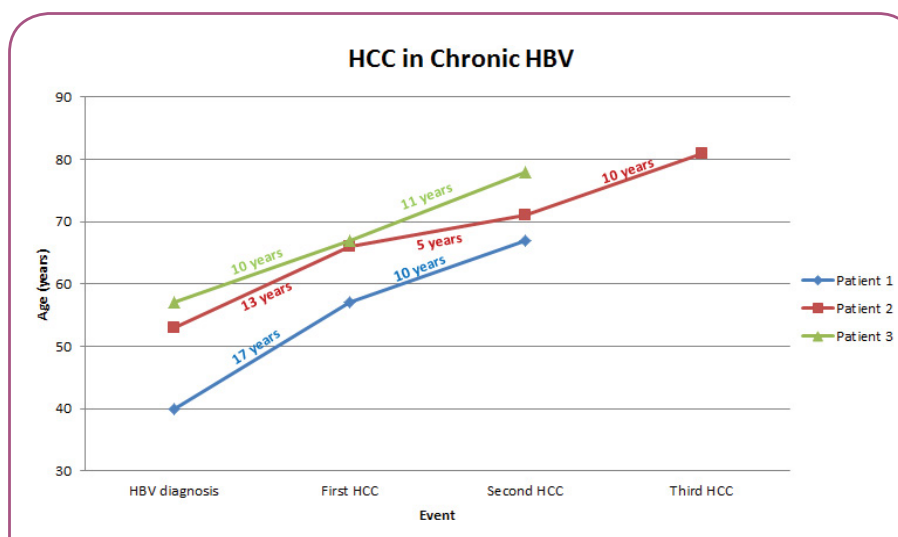

Figure 4 Three patient cases demonstrating the long duration time between different HCC diagnoses.

into a covalently closed circular DNA (cccDNA) in the host cell nucleus. However, ten percent of the virions contain doublestranded linear DNA that becomes integrated into the host DNA. Although integration into the host DNA is not required for HBV replication, it is likely a key step in hepatocarcinogenesis [20-22]. Anti-HBV therapies such as lamivudine, tenofovir and entecavir inhibit HBV-DNA polymerase which suppresses viral replication [3-6]. However, these medications do not inhibit integration and therefore do not impact subsequent HCC development. This key step in hepatocarcinogenesis may represent an innovative pathway to target in future drug development. In addition to viral DNA integration, there are several other steps in the HBV life cycle that can potentially serve as targets for drug development and curative therapies in the future [18-24]. Currently there are several steps in the HBV life cycle that are not completely understood such as mechanism of entry, cccDNA formation and regulation, intracellular trafficking and morphogenesis and interaction with the host immune system. Further information specifically regarding HBV cccDNA is needed as its persistence in the hepatocytes and integration into the host genome prevents its eradication. In addition to information about cccDNA, more understanding is needed regarding viral entry into newly formed hepatocytes and interactions between the virus and host immune system as these could serve as targets for future curative therapies. Another target for future therapy is inhibition of maturation of the pregenomic mRNA in the hepatocyte nucleus and subsequent blockage of export to hepatocyte cytoplasm. Inhibiting this process would reduce the generation of HBV core particle in the cytoplasm and significantly reduce the production of cccDNA [19-24]. While we wait for such medications, it is important to understand that patients on anti-HBV therapy with undetectable levels of HBV are still at increased risk for HCC development. Given the complexity of HBV replication, developing a curative therapy is not easy. Multiple steps exist that can and should be targeted such as the following: entry of the virus by blocking the receptors on hepatocytes, replication of new HBV DNA from the pregenomic RNA (has been successful in this aspect with currently available nucleos(t)ide analogues) and enhanced host innate immunity (such as small molecule agonists of toll-like receptors). In addition, development of a therapeutic vaccine engineered to activate an $\mathrm{HBV}$-specific $\mathrm{T}$ cell immune 
response to directly target cccDNA in addition to several others could also have the potential to be a successful curative therapy. While the anti-HBV therapies do not provide a cure for HBV nor prevent HCC development, there is no question regarding the improved survival and quality of life they provide in patients with chronic HBV. Even in patients with recurrent HCC, improved liver function and reversal of fibrosis have been seen and are attributed to the use of antiviral medications. [1-10] While these cases demonstrate the persistent risk for HCC, it is important to remember the strides that have been made in medication and vaccine development and the impact antiviral medications have had on patients with chronic HBV. It is also important to support research aimed at finding a cure for chronic $\mathrm{HBV}$ in an effort to eradicate the persistent risk for HCC seen in these patients.

\section{References}

1 Beasley RP, Lin CC, Hwang LY, Chien CS (1981) Hepatocellular carcinoma and hepatitis B virus: A prospective study of 22707 men in Taiwan. The Lancet 318: 1129-1133.

2 Chen CJ, Yang HI, Su J, Jen CL, You SL, et al. (2006) Risk of hepatocellular carcinoma across a biological gradient of serum hepatitis B virus DNA level. JAMA 295: 65-73.

3 Liaw YF, Sung JY, Chow WC, Farrell G, Lee CZ, et al. (2004) Lamivudine for patients with chronic hepatitis $\mathrm{B}$ and advanced liver disease. $\mathrm{N}$ Engl J Med 351: 1521-1531.

4 Eun JR, Lee HJ, Kim TN, Lee KS (2010) Risk assessment for the development of hepatocellular carcinoma: according to ontreatment viral response during long-term lamivudine therapy in hepatitis B virus related liver disease. J Hepatol 53: 118-125.

5 Hosaka T, Suzuki F, Kobayashi M, Seko Y, Kawamura Y, et al. (2013) Long-term entecavir treatment reduces hepatocellular carcinoma incidence in patients with hepatitis B virus infection. Hepatology 58: 98-107.

6 Kim WR, Loomba R, Berg T, Yee LJ, Dinh PV, et al. (2015) Impact of long-term tenofovir disoproxil fumarate on incidence of hepatocellular carcinoma in patients with chronic hepatitis B. Cancer 121: 3631-3638.

7 Papatheodoridis GV, Lampertico P, Manolakopoulos S, Lok A (2010) Incidence of hepatocellular carcinoma in chronic hepatitis $B$ patients receiving nucleos(t)ide therapy: A systematic review. J Hepatol 53: 348-356.

8 Papatheodoridis GV, Manolakopoulos S, Touloumi G, Vourli G, Raptopoulou-Gigi M, et al. (2011) Virological suppression does not prevent the development of hepatocellular carcinoma in HBeAgnegative chronic hepatitis $B$ patients with cirrhosis receiving oral antiviral(s) starting with lamivudine monotherapy: results of the nationwide HEPNET. Greece cohort study. Gut 60: 1109-1116.

9 Vlachogiannakos J, Papatheodoridis G (2013) Hepatocellular carcinoma in chronic hepatitis B patients under antiviral therapy. World J Gastroenterol 19: 8822-8830.

10 Ando $\mathrm{Y}$, Ishigami M, Ishizu $\mathrm{Y}$, Kuzuya T, Honda T, et al. (2018) Cumulative incidence and risk factors for the development of hepatocellular carcinoma in patients with chronic hepatitis B who achieved sustained disappearance of viremia by nucleos(t)ide analogue treatment. Hepatol Res 48: 240-25.
Recently, the progress made in the development of HBV curative therapies was updated by Levrero et al. [23] and Block et al. [24].

\section{Conclusion}

The risk of HCC persists in patients who maintain successful HBV suppression for years with antiviral therapy. Current antiviral therapy (nucleos(t)ide analogues) can suppress viral replication but is unable to eliminate the cccDNA in the nucleus of the hepatocytes. There is a need for HBV cure drugs.

\section{Conflict of Interest}

The authors declare that they have no conflict of interest that competes with any of the contents of the manuscript.

11 Dargan A, Wong SY, Coben R, Conn M, Dimarino AJ, et al. (2017) Persistent risk for hepatocellular carcinoma after more than a decade of successful hepatitis B virus suppression. Minerva Gastroenterol Dietol 63: 74-76.

12 Yoo J, Hann HW, Coben R, Conn M, DiMarino AJ (2018) Update treatment for HBV infection and persistent risk for hepatocellular carcinoma: prospect for an HBV cure. Diseases 6: 27.

13 Kubo S, Tanaka H, Takenmura S, Yamamoto S, Hai S, et al. (2007) Effects of lamivudine on outcome after liver resection for hepatocellular carcinoma in patients with active replication of hepatitis $B$ virus. Hepatol Res 37: 94-100.

14 Huang G, Lau WY, Wang ZG, Pan ZY, Yuan SX, et al. (2015) Antiviral therapy improves postoperative survival in patients with hepatocellular carcinoma: a randomized controlled trial. Ann Surg 261: 56-66.

15 Yin J, Li N, Han Y, Xue J, Deng Y, et al. (2013) Effect of antiviral treatment with nucleotide/nucleoside analogs on postoperative prognosis of hepatitis B virus-related hepatocellular carcinoma: a two-stage longitudinal clinical study. J Clin Oncol 31: 3647-3655.

16 Chuma M, Hige S, Kamiyama T, Meguro T, Nagasaka A, et al. (2009) The influence of hepatitis B DNA level and antiviral therapy on recurrence Canadian Journal of Gastroenterology and Hepatology 11 after initial curative treatment in patients with hepatocellular carcinoma. J Gastroenterol 44: 991-999.

17 Wu CY, Chen YJ, Ho HJ, Hsu YC, Kuo KN, et al. (2012) Association between nucleoside analogues and risk of hepatitis $\mathrm{B}$ virus-related hepatocellular carcinoma recurrence following liver resection. JAMA 308: 1906-1913.

18 Hann HW, Coben R, Brown D, Needleman L, Rosato E, et al. (2014) A long-term study of the effects of antiviral therapy on survival of patients with HBV associated hepatocellular carcinoma (HCC) following local tumor ablation. Cancer Medicine 3: 390-396.

19 Summers J, Mason WS (1982) Replication of the genome of a hepatitis B-like virus by reverse transcription of an RNA intermediate. Cell 29: 403-415.

20 Shafritz DA, Shouval D, Sherman HI, Hadziyannis SJ, Kew MC (1981) Integration of hepatitis B virus DNA into the genome of liver cells in chronic liver disease and hepatocellular carcinoma: Studies in percutaneous liver biopsies and post-mortem tissue specimens. N Engl J Med 305: 1067-1073. 
21 Ganem D, Prince AM (2004) Hepatitis B Virus Infection - Natural history and clinical consequences. N Engl J Med 350: 1118-1129.

22 Zeisel MB, Lucifora J, Mason WS, Sureau C, Beck J, et al. (2015) Towards an HBV cure: A state-of-the-art and unresolved questionsreport of the ANRS workshop on HBV cure. Gut 64: 1314-1326.
23 Levrero M, Testoni B, Zoulim F (2016) HBV cure: why, how, when? Curr Opin Virol 18: 135-143.

24 Block TM, Alterc H, Browna N, Brownstein A, Brosgart C, et al. (2018) Research priorities for the discovery of a cure for chronic hepatitis $B$ : Report of a workshop. Antivir Res 150: 93-100. 